\title{
Actin coating and compression of fused secretory vesicles are essential for surfactant secretion - a role for Rho, formins and myosin II
}

\author{
Pika Miklavc ${ }^{1}$, Elena Hecht ${ }^{2}$, Nina Hobi ${ }^{3}$, Oliver H. Wittekindt ${ }^{1}$, Paul Dietl ${ }^{1}$, Christine Kranz $^{2}$ and Manfred Frick ${ }^{1, *}$ \\ ${ }^{1}$ Department of General Physiology, University of Ulm, Albert-Einstein Allee 11, $89081 \mathrm{UIm}$, Germany \\ ${ }^{2}$ Institute of Analytical and Bioanalytical Chemistry, University of Ulm, Albert-Einstein Allee 11, 89081 Ulm, Germany \\ ${ }^{3}$ Department of Physiology and Medical Physics, Innsbruck Medical University, Fritz-Pregl-Strasse 3, A-6020 Innsbruck, Austria \\ *Author for correspondence (manfred.frick@uni-ulm.de)
}

Accepted 3 February 2012

Journal of Cell Science 125, 2765-2774

(C) 2012. Published by The Company of Biologists Ltd

doi: 10.1242/jcs. 105262

\section{Summary}

Secretion of vesicular contents by exocytosis is a fundamental cellular process. Increasing evidence suggests that post-fusion events play an important role in determining the composition and quantity of the secretory output. In particular, regulation of fusion pore dilation and closure is considered a key regulator of the post-fusion phase. However, depending on the nature of the cargo, additional mechanisms might be essential to facilitate effective release. We have recently described that in alveolar type II (ATII) cells, lamellar bodies (LBs), which are secretory vesicles that store lung surfactant, are coated with actin following fusion with the plasma membrane. Surfactant, a lipoprotein complex, does not readily diffuse out of fused LBs following opening and dilation of the fusion pore. Using fluorescence microscopy, atomic force microscopy and biochemical assays, we present evidence that actin coating and subsequent contraction of the actin coat is essential to facilitate surfactant secretion. Latrunculin B prevents actin coating of fused LBs and inhibits surfactant secretion almost completely. Simultaneous imaging of the vesicle membrane and the actin coat revealed that contraction of the actin coat compresses the vesicle following fusion. This leads to active extrusion of vesicle contents. Initial actin coating of fused vesicles is dependent on activation of Rho and formin-dependent actin nucleation. Actin coat contraction is facilitated by myosin II. In summary, our data suggest that fusion pore opening and dilation itself is not sufficient for release of bulky vesicle cargos and that active extrusion mechanisms are required.

Key words: Actin, Exocytosis, Formin, Lamellar body, Myosin, Rho

\section{Introduction}

Regulated secretion is a fundamental process in many different types of eukaryotic cells. In general, vesicle contents are released by exocytosis of secretory vesicles. During exocytosis, a sequence of highly regulated steps leads to fusion of exocytic vesicles with the plasma membrane (PM), opening of a fusion pore and finally content release (Bean et al., 1994; Lindau and Gomperts, 1991; Rettig and Neher, 2002; Südhof, 2004). In the classical model, the initial fusion pore dilates until the membrane is completely collapsed into the PM and the entire vesicle content is passively released in an all-or-none manner ('full fusion') (Heuser and Reese, 1973; Südhof, 2004). However, at least for large secretory granules, the classical view of instantaneous cargo release and vesicle collapse is increasingly being challenged. Evidence suggests that in non-neuronal cell types, vesicle content release is regulated during the post-fusion phase. In this 'kissand-run' model, the vesicle does not readily collapse into the PM and vesicle content release is regulated by opening and closure of a dynamic fusion pore before intact vesicles are retrieved from the PM ('cavicapture') (Rizzoli and Jahn, 2007; Soekmadji and Thorn, 2010). The duration of the fusion pore open state and its diameter depend on stimulation (Vardjan et al., 2007), with stable fusion pore diameters also depending on the diameter of the fused vesicles (Jorgačevski et al., 2010). A range of factors, such as
$\mathrm{Ca}^{2+}$ (Haller et al., 2001a), myosin II (Bhat and Thorn, 2009), Munc-18 (Jorgačevski et al., 2011), dynamin (Anantharam et al., 2011) and F-actin (Larina et al., 2007) amongst others (Jackson and Chapman, 2008), have been suggested as molecular mediators for fusion pore transitions. The actual release of vesicle contents is generally thought to be a rather passive process, with vesicle contents diffusing through open fusion pores. This is easily conceivable for soluble vesicle contents such as neurotransmitters, hormones and some proteins. However, release of non-soluble, bulky vesicle contents or granule matrices is unlikely to happen in such a passive manner.

ATII cells secrete pulmonary surfactant by exocytosis of lamellar bodies (LBs). Surfactant, a bulky complex made up of tightly packed lipids and proteins, has properties that justify it as a solid state matter. The lipophilic nature of surfactant impedes rapid dissolution and dispersal in aqueous solution and hence surfactant does not readily diffuse out of fused LBs following opening of the exocytic fusion pore. Rather, surfactant is so insoluble, that it might remain entrapped within the fused vesicle for many minutes after the onset of exocytosis and the fusion pore apparently acts as a mechanical barrier for the release of the large, macromolecular vesicle cargo entity, which is released as one single complex as observed by live-cell microscopy (Dietl and Haller, 2005; Dietl et al., 2001; Haller et al., 2001a; Singer 
et al., 2003). Upon fusion of the LB with the PM, fusion pore expansion in these cells is slow (Haller et al., 2001a; Singer et al., 2003). Hence, it is conceivable that secretion of surfactant is not merely a passive process, but requires additional mechanisms to be effective. Almost three decades ago, transmission electron micrographs of fused LBs suggested that actin is involved in expulsion (Tsilibary and Williams, 1983a; Tsilibary and Williams, 1983b) and immunostaining experiments confirmed that F-actin encloses individual LBs in proximity to the PM (van Weeren et al., 2004). We have recently shown that LBs are coated with actin following fusion with the PM (Miklavc et al., 2009b).

Actin coating of secretory vesicles has been shown in a variety of cell types and various functions for the actin coating were proposed. In zebrafish oocytes, actin reorganization after fusion was considered to facilitate retrieval of evacuated granules (Becker and Hart, 1999). Similarly, in Xenopus oocytes, actin coating of cortical granules was observed following fusion. In these cells 'specific' actin coating of fused vesicles is achieved by membrane-fusion-dependent compartment mixing ( $\mathrm{Yu}$ and Bement, 2007a). Upon fusion, key components of the PM can diffuse into the fused secretory granule membrane and act as trigger for local actin assembly ('kiss-and-coat') (Sokac and Bement, 2006). Actin coating was postulated to stabilize fused cortical granules for compensatory endocytosis, but possibly also to facilitate content release (Sokac et al., 2003). In pancreatic acinar cells, actin coating was proposed to stabilize fused zymogen granules during exocytosis (Nemoto et al., 2004) and maintain an open fusion pore (Larina et al., 2007), although it is not quite clear whether actin coating of zymogen granules starts during the pre-fusion phase or following fusion (Turvey and Thorn, 2004; Valentijn et al., 2000). Therefore, actin coating might also play a role for the pre-fusion phase of exocytosis (Eitzen, 2003; Gasman et al., 2004). Recent data from in vivo imaging experiments suggest that actin coating of fused vesicles provides a scaffold to facilitate the collapse of vesicles into the PM (Masedunskas et al., 2011).

The 'kiss-and-coat' mechanism, involving actin coating of fused vesicles, is uniquely suited to promote discharge of secretory material under conditions where it cannot be released rapidly (Sokac and Bement, 2006). However, only recently, evidence emerged that actin coating of fused vesicles plays an active role in the release of vesicle content (Nightingale et al., 2011). We also postulated that in ATII cells, actin coating of fused LBs facilitates surfactant release (Miklavc et al., 2009b). However, it remained open as to whether actin coating is essential for surfactant release, how the force for expulsion is generated and also, how actin coating of LBs upon fusion is triggered and regulated. In this study, we present evidence that actin coating of fused vesicles and subsequent contraction of the actin coat are essential for the release of surfactant. Contraction of the actin coat compresses fused LBs and promotes expulsion of surfactant. Contraction is assisted by myosin II. Inhibiting formation of the actin coat prevents compression of fused LBs and hence almost entirely restrains surfactant secretion. Furthermore, we demonstrate that initial recruitment of actin to LBs upon fusion with the PM depends on active Rho GTPases and that formins probably play a role in actin nucleation. In summary, our data suggest that simply opening a door (fusion pore) is not sufficient for efficient release of bulky vesicle cargos and that active extrusion mechanisms are required.

\section{Results}

\section{Actin coating of fused LBs is essential for surfactant expulsion and efficient release}

We have recently reported actin coating of LBs upon fusion with the PM and postulated a role for this in surfactant release (Miklavc et al., 2009b) (supplementary material Movie 1). Actin coat formation probably depends on de novo nucleation of actin on LBs following fusion (Miklavc et al., 2009b) and can be inhibited by latrunculin B (LatB, $10 \mu \mathrm{M}$ ) or jasplakinolide $(1 \mu \mathrm{M})$ treatment (Fig. 1). Here, we investigated whether surfactant expulsion and hence secretion depends on actin coat formation.

We incubated ATII cells with fluorescent phosphatidylcholine (BODIPY-PC) overnight to metabolically label surfactant. BODIPY-PC incorporates into surfactant (Ravasio et al., 2011). At the beginning of the experiment, cells were stimulated with $100 \mu \mathrm{M}$ ATP, a potent agonist for LB fusion and surfactant secretion (Frick et al., 2001). LB fusions with the PM were indicated by rapid decay of Lysotracker Red (LTR) fluorescence in fused vesicles (Haller et al., 2001a; Miklavc et al., 2011) (see also Fig. 1). Analysis of the onset of fluorescence decay of LTR,
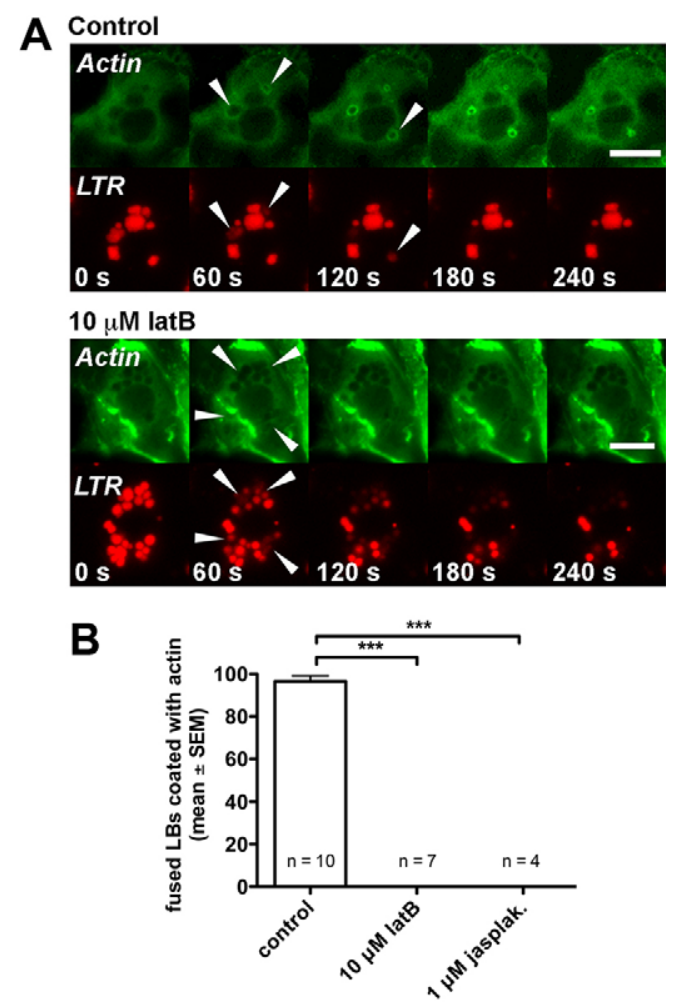

Fig. 1. Actin coating of fused vesicles is inhibited by LatB and jasplakinolide. (A) Simultaneous imaging of actin--GFP (green) and LTR (red) reveals actin coating of vesicles upon fusion under control conditions (top panels). The presence of $10 \mu \mathrm{M}$ LatB completely inhibits actin coat formation following LB fusion with the PM (bottom panels). Arrowheads indicate individual fused vesicles, revealed by a decrease in vesicular LTR fluorescence due to diffusion of LTR from the vesicle lumen. Cells were stimulated with $100 \mu \mathrm{M}$ ATP at $t=10$ seconds. Scale bars: $10 \mu \mathrm{m}$.

(B) Treatment of ATII cells with either $10 \mu \mathrm{M}$ LatB or $1 \mu \mathrm{M}$ jasplakinolide completely inhibits actin coating of fused vesicles. $n$ represents number of experiments for each condition, up to 26 fusions were analyzed in each experiment. $* * * P<0.005$. 
indicating fusion pore opening, and BODIPY-PC, indicating surfactant expulsion, in individual vesicles, clearly revealed a substantial delay between fusion pore opening and BODIPY-PC release (78.92 \pm 11.52 seconds, $n=25)$ (Fig. 2A,B). This indicated that BODIPY-PC did not readily diffuse from fused vesicles. Moreover, with most fusions $(91.53 \pm 5.42 \%, n=29$, six experiments), we observed protrusions of BODIPY-PC from fused LBs once fluorescence of BODIPY-PC decreased within the fused LB (Fig. 2A,D; supplementary material Movie 2). This probably represents expulsion of surfactant from fused LBs. When actin coat formation was inhibited by pre-treatment of ATII cells with $10 \mu \mathrm{M}$ LatB, see (Fig. 1), BODIPY-PC fluorescence did not decrease during the time of the experiment (up to 10 minutes after fusion, Fig. 2C). Also, significantly fewer protrusions could be observed $(1.92 \pm 1.92 \%, n=30$ fusions, four experiments, $P<0.0001$, Fig. 2D). This indicates that most of surfactant remains inside fused vesicles and supports our hypothesis that actin coat compression is essential for efficient surfactant secretion.

To rule out a potential impact of excess BODIPY-PC incorporation on surfactant properties and release, we also used a modified FRAP assay to determine whether surfactant is expelled from fused LBs. The assay is based on the fluorescent properties of FM1-43, a fluorescent dye that is essentially nonfluorescent in aqueous solutions but yields a bright signal when incorporated into surfactant (Haller et al., 2001a; Haller et al., 1998). FM1-43 cannot pass the PM, but when LBs fuse with the PM, FM1-43 can incorporate into surfactant, resulting in an intense localized fluorescence signal. FM1-43 fluorescence of fused vesicles was bleached 3-5 minutes after fusion, a time when, according to the results from the BODIPY-PC experiments, most LBs have expelled their content under control conditions. Subsequently, recovery of FM1-43 fluorescence due to incorporation of non-bleached FM1-43 molecules was monitored (Fig. 3A,B). Recovery of FM1-43 fluorescence was significantly slower for LBs fused in the presence of LatB $\left(t_{1 / 2}=131.70 \pm 9.55\right.$ seconds, $\left.n=62\right)$ than under control conditions $\left(t_{1 / 2}=90.62 \pm 13.27\right.$ seconds, $\left.n=57, P=0.01\right)$. In our model, the accelerated recovery of FM1-43 fluorescence under control conditions is due to enhanced access of FM1-43 to at least partially expelled surfactant. In LatB-treated cells, most surfactant will be retained inside fused vesicles and access of FM1-43 is restricted by a narrow fusion pore (Fig. 3D) (Haller et al., 2001a). To test this hypothesis, we also analyzed FRAP recovery of fully released or expelled surfactant particles (with no restriction for FM1-43 diffusion). FM1-43 recovery of fully released surfactant particles was slightly, but not significantly, faster than the recovery observed in LBs fused under control conditions $\left(t_{1 / 2}=51.64 \pm 6.65\right.$ seconds, $\left.n=24, P=0.07\right)$. The even faster recovery is probably due to the absence of any restriction (i.e. fusion pore, LB limiting membrane) for FM1-43 incorporation into surfactant in these particles. Analysis of the frequency distribution of half-times of recovery confirmed that FM1-43 recovery in fused $\mathrm{LBs}$ is slower when actin coat formation and compression are inhibited. Half-times for FM1-43 recovery are $<75$ seconds for the majority of control and released LBs $(73.7 \%$ and $87.5 \%$, respectively). By contrast, only $27.4 \%$ of fused LBs had half-times of recovery $<75$ seconds after LatB treatment (Fig. 3C).

Both the BODIPY-PC expulsion assay and the FM1-43 FRAP assay on fused vesicles, provide strong evidence that actin coating of fused LBs is essential for effective release of surfactant. Full or partial expulsion of surfactant was also observed in a complementary experiment, when fused vesicles were investigated by AFM. AFM images of non-stimulated ATII cells showed regularly shaped, micrometer-sized protrusions

A

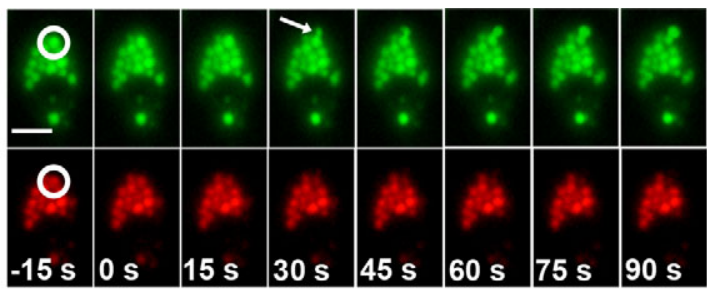

B
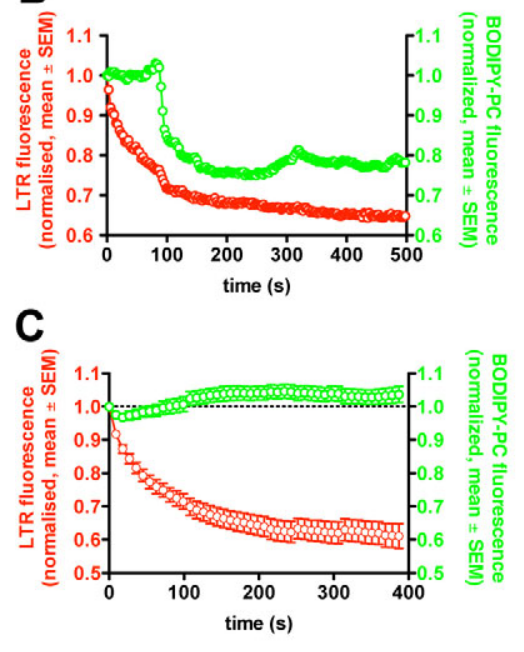

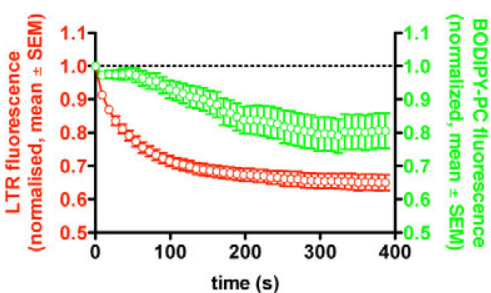

D

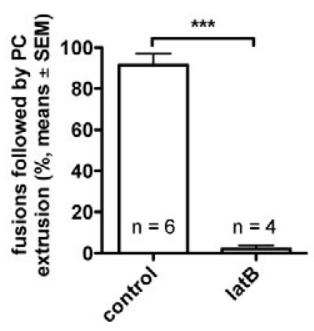

Fig. 2. LatB inhibits squeezing of surfactant from LBs following fusion. (A) Simultaneous imaging of BODIPY-PC (top row) and LTR (bottom row). Onset of LTR fluorescence decrease denotes time of fusion $(0 \mathrm{~s})$. Expulsion of surfactant is clearly delayed as seen by onset of BODIPY-PC extrusion (arrow) from fused vesicle. Changes of mean fluorescent intensities were analyzed in circular region of interest (circle). Time stamps indicate time related to LB fusion. Scale bar: $10 \mu \mathrm{m}$. (B) Left: time course of LTR (red) and BODIPY-PC (green) fluorescence following LB fusion within the area of a fused LB as illustrated in A. Right: means of 21 fused LBs, the average delay between LB fusion and BODIPY-PC extrusion is $78.92 \pm 11.52$ seconds. (C) Time course of LTR (red) and BODIPY$\mathrm{PC}$ (green) fluorescence following LB fusion in LatB-treated cells. BODIPY-PC remains within the LB for extended periods following fusion. Data are from 31 individual LB fusions. (D) Percentage of LB fusions followed by extrusion of BODIPY-PC as detected in A. LatB abolishes BODIPY-PC extrusion following LB fusion. $n$ represents number of experiments for each condition, up to 13 fusions were analyzed in each experiment. ${ }^{* * *} P<0.005$. 
A

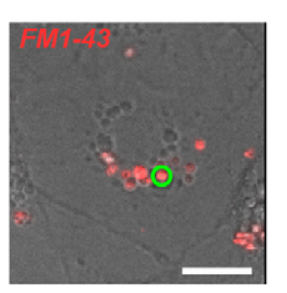

B
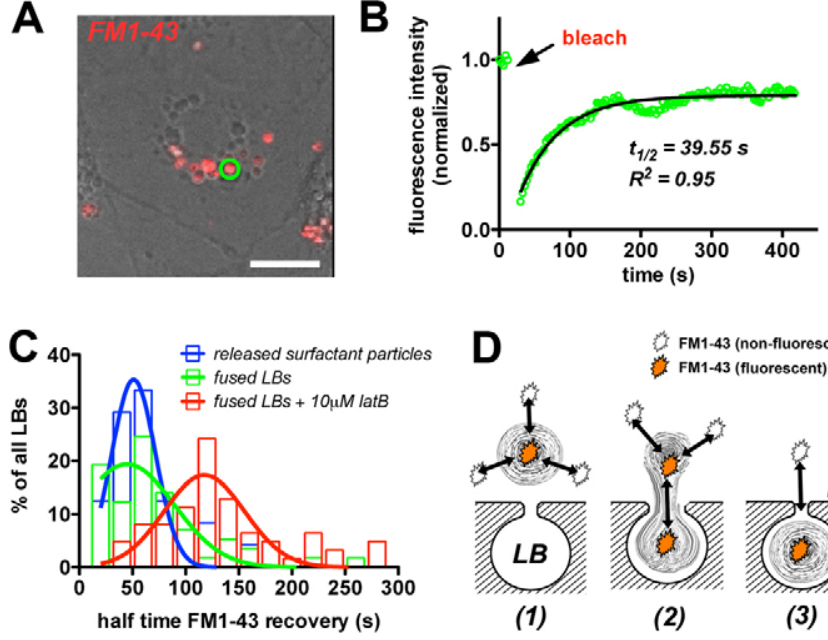

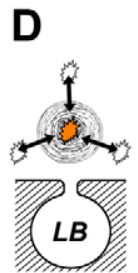

(1)

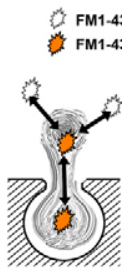

(2) FM1-43 (non-fluorescent)
FM1-43 (fluorescent)

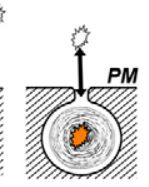

(3)

Fig. 3. FRAP confirms surfactant extrusion from LBs upon fusion. (A) Overlay of transmission and FM1-43 fluorescence image of ATII cells. Upon fusion with the PM LBs exhibit bright FM1-43 fluorescence (red). The green circle denotes the area of a single LB that was bleached 5 minutes after fusion. Scale bar: $10 \mu \mathrm{m}$. (B) Time course of the FM1-43 fluorescence within the area of the green circle in A. Bleaching occurred at $t=15$ seconds. Analysis of the FM1-43 fluorescence recovery was performed by calculating the ratio between mean fluorescence intensity of the bleached LB and the mean fluorescence intensity of an unbleached LB to correct for background bleaching during pre- and post-bleach acquisition. Ratio values were then expressed as a percentage of the pre-bleach ratio value. These normalized data were fitted to a single exponential curve (black line) for calculation of $t_{1 / 2}$. (C) Frequency distribution for half times of FM1-43 fluorescence recovery after bleaching. Recovery of FM1-43 fluorescence in surfactant of fused LBs is slightly slower than in fully released particles, however recovery in LBs is significantly slower in the presence of LatB. Data are from 24-62 vesicles per condition. (D) Model for recovery of FM1-43 fluorescence after bleaching based on data from C. Diffusion of FM1-43 into fully released surfactant particles (1) is almost unrestricted. (2) LBs fused under control condition, where surfactant is (at least) partially expelled and (3) LBs fused in LatBtreated cells, surfactant is trapped inside fused LBs when actin coating and compression are inhibited.

(Fig. 4A, visible as small bumps), which are indicative of LBs residing beneath the PM around the nucleus (Hecht et al., 2011). The overlay of the bright field with the FM1-43 fluorescence image confirmed that these LBs have not yet fused (Fig. 4A). However, when probing the cells 5-15 minutes after stimulation, we regularly found that FM1-43-positive structures, indicating fused LBs, were significantly more pronounced. One explanation therefore might be that LBs are in even closer proximity to the PM following fusion. Alternatively, this could be indicative of full or partially expelled surfactant sticking to the cell surface at sites of fused LBs. This is supported by our observation that under control conditions, $>60 \%$ of these FM1-43-positive structures $(n=8)$ were pushed away by the AFM tip in low force contact as well as dynamic (AAC) mode (Fig. 4B; supplementary material Movie 3). Surfactant is a very 'sticky' substance that can easily adhere to the AFM tip when scanning across fused, surfactant-releasing LBs (Hecht et al., 2011). Once FM1-43-labelled surfactant has been pushed away, we observed planar areas at the sites of the fused vesicles (Fig. 4C) suggesting that the fused LBs have been completely compressed or collapsed into the PM during surfactant release. Congruently, when

A

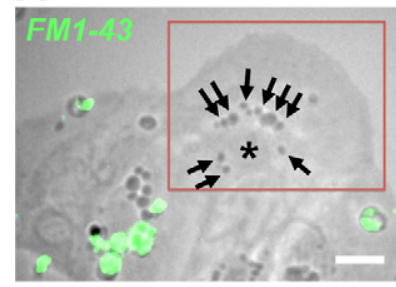

B

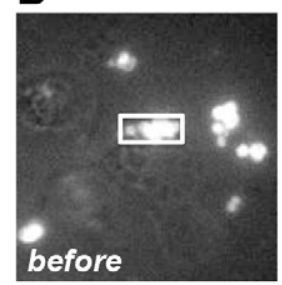

D

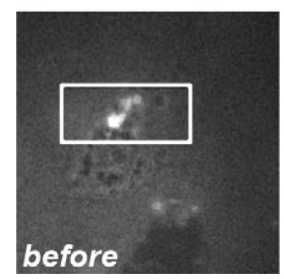

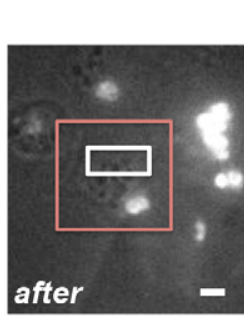

C

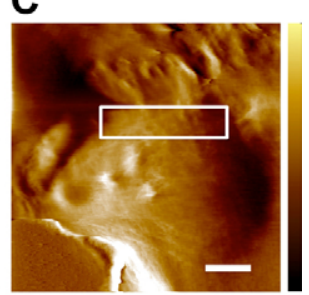

E

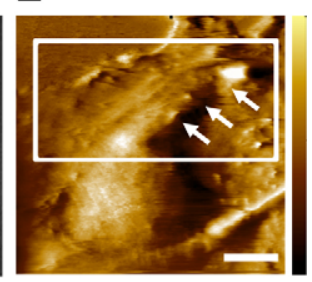

Fig. 4. AFM data reveal extracellular localization of surfactant following LB fusion. (A) Left panel is an overlay of transmission and FM1-43 fluorescence image of ATII cells. Black arrows indicate intracellular LBs that have not yet fused. LBs exhibit bright FM1-43 fluorescence (green) following fusion. Right panel shows AFM deflection image of the red boxed area in the left image. Non-fused LBs, residing beneath the PM, are clearly seen as micrometer-sized protrusions (white arrows) around the nucleus (asterisk). (B) Overlay of transmission and FM1-43 fluorescence images of ATII cells following stimulation. Left: surfactant of fused vesicles is brightly stained by FM1-43 (white structures) before imaging the area in the white box by AFM. Right: image taken immediately after scanning the area in the white box by AFM. FM1-43-labelled structures have been pushed away by the AFM tip, see also supplementary material Movie 3. (C) AFM deflection image of the red boxed area in B. The area where FM1-43 labelled surfactant has been pushed away during AFM scanning (white box) appears planar, typical protrusions from LBs are absent. (D) As for B, but cells are treated with $10 \mu \mathrm{M}$ LatB. In LatB-treated cells FM1-43-stained-surfactant is not pushed away by the AFM tip during scanning. (E) AFM deflection image of the red boxed area in D recorded after AFM imaging. Micrometer-sized protrusions (white arrows) can be seen where FM1-43 stained surfactant is visible in D, indicating that FM1-43-stained surfactant is still entrapped within fused LBs and could not be pushed away by the AFM tip during scanning. Scale bars: $5 \mu \mathrm{m}$.

scanning LatB-treated ATII cells, FM1-43-labelled surfactant was never pushed away by the AFM tip as observed under control conditions ( $n=4$, Fig. 4D; supplementary material Movie 4). This again supports our hypothesis that surfactant remains trapped inside LBs following fusion if actin coating is inhibited. If this is the case then rates of surfactant secretion will also be reduced when actin coat formation is inhibited.

We next analyzed the amount of secreted phosphatidylcholine (PC, the main component of surfactant) following stimulation using a recently described enzymatic protocol (Garcia-Verdugo et al., 2008). 15 minutes after stimulation with $100 \mu \mathrm{M}$ ATP, the PC content of supernatants $(1.45 \pm 0.32 \mu \mathrm{M}, n=4)$ was 
significantly enhanced compared with non-stimulated cells $(0.47 \pm 0.06 \mu \mathrm{M}, \quad n=4, \quad P=0.04), \quad$ LatB treatment almost completely impeded PC secretion following stimulation $(0.79 \pm 0.13 \mu \mathrm{M}, n=4)$ (Fig. 5). In summary, all of these results confirm an essential role for actin in secretion of surfactant.

\section{Fused LBs are compressed by actin coat contraction}

Actin coating of fused vesicles has been observed in several cell types and various biological functions have been proposed (Becker and Hart, 1999; Eitzen, 2003; Gasman et al., 2004; Nemoto et al., 2004; Sokac et al., 2003; Valentijn et al., 2000) including an 'active' role in vesicle content release (Nightingale et al., 2011). However, the mechanisms by which this occurs are largely unclear. In particular, it is not known whether the observed contraction of the actin coat directly compresses the fused vesicle. Direct and simultaneous observations of the actin coat and the vesicle membrane are not available (Schafer, 2003).

To investigate whether the vesicle itself is compressed upon actin coat contraction, we co-transfected cells with a marker for the LB membrane (lamp3-GFP) (Albrecht et al., 2010) and actin-DsRed and analyzed changes in vesicle and actin coat perimeter following LB fusion with the PM. These experiments revealed a clear correlation between contraction of the actin coat and LB size. LBs were shrinking with increasing actin coat contraction (Fig. 6A,C). Lamp3-GFP slowly diffused from the LB membrane into the PM following fusion, making analysis difficult at times $>60$ seconds following fusion. Therefore, in a complementary approach, we used a marker for the PM (lynDsRed) (Frick et al., 2007) that rapidly diffuses into the LB membrane following fusion, resulting in a strong signal for extended periods (Fig. 6B,D; supplementary material Movie 5). These experiments confirmed the correlation between actin coat contraction and LB compression as observed with lamp3-GFP. When actin coat formation was inhibited with LatB, vesicles did not change in size following fusion (Fig. 6E). These results clearly indicate that the actin coat contraction leads to compression of fused LBs.

\section{Myosin II facilitates actin coat contraction}

We next tested whether myosins play a role in the observed contraction of the actin coat, generating force for 'active' vesicle

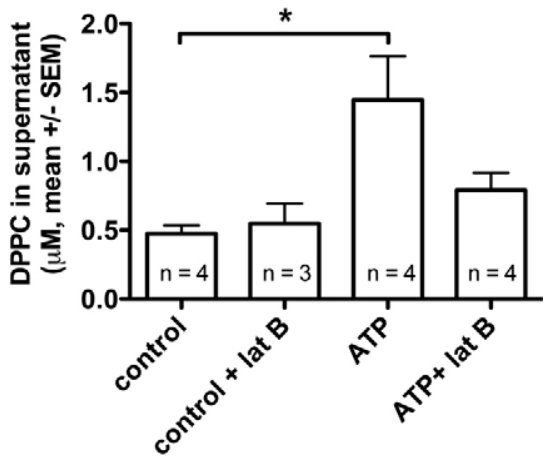

Fig. 5. Inhibition of actin coat formation inhibits surfactant secretion. DPPC concentration in supernatant as a measure of surfactant secretion was significantly increased $15 \mathrm{~min}$ after stimulation of LB exocytosis with $100 \mu \mathrm{M}$ ATP compared with non-stimulated cells (control). Treatment with $10 \mu \mathrm{M}$ LatB almost completely inhibits stimulation dependent DPPC secretion. $n$ represents number of experiments for each condition. ${ }^{*} P<0.05$.
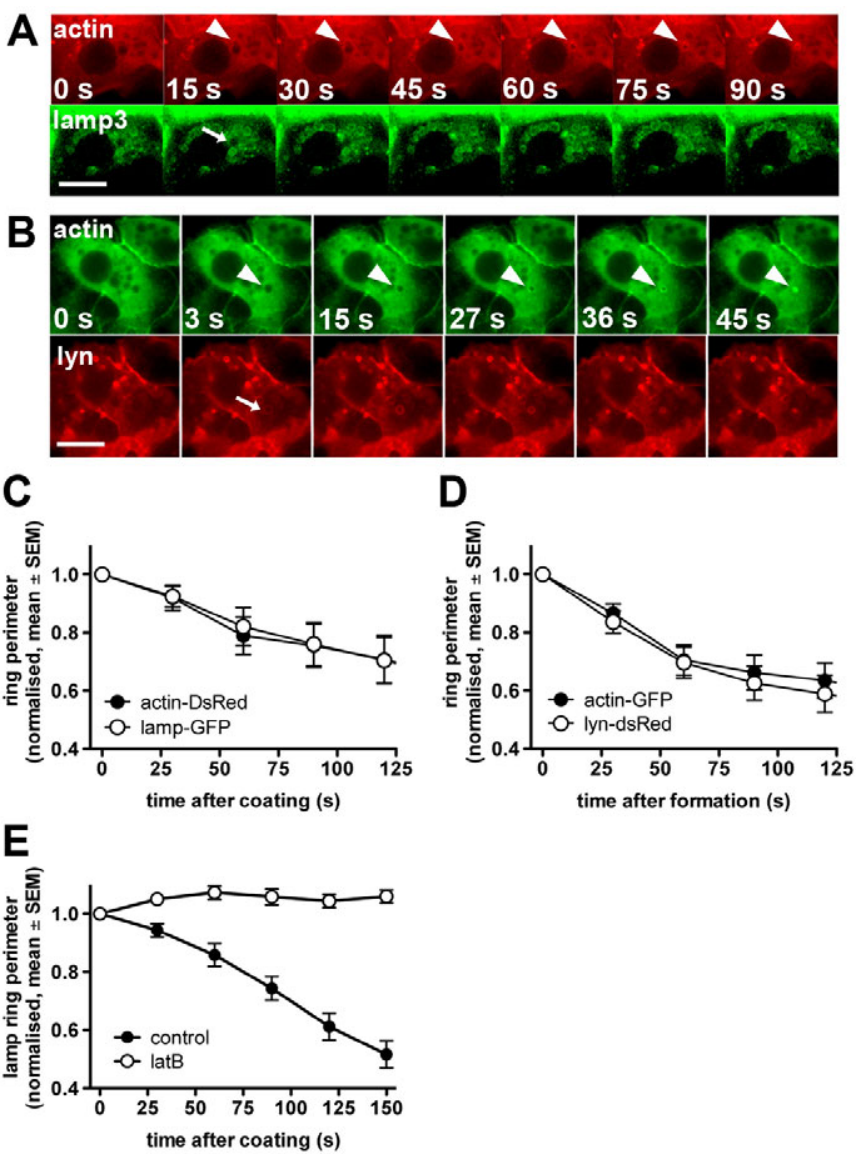

Fig. 6. LBs are compressed by actin coats. (A) Simultaneous imaging of actin-DsRed (top row) and LB membrane marker lamp3-GFP (bottom row) A LB (arrow) is compressed at the same time as the actin coat (arrowhead) contracts, as indicated by decrease in LB membrane perimeter. Time stamps indicate time after fusion. Scale bar: $10 \mu \mathrm{m}$. (B) Simultaneous imaging of actin-GFP (top row) and membrane marker lyn-DsRed (lower row). LynDsRed rapidly diffuses into a LB after fusion (arrow). The lyn-DsRedlabelled LB is compressed at the same time as the actin coat contracts (arrowheads), as indicated by decrease in LB membrane perimeter. Time stamps indicate time after fusion. Scale bar: $10 \mu \mathrm{m}$. (C,D) The decrease of the LB perimeter, indicated by LB membrane marker lamp3-GFP (C) or membrane marker lyn-DsRed (D), correlates with the contraction of the actin coat. Means are from 13 and 16 LBs, respectively. (E) Compression of LBs following fusion is inhibited when actin polymerization is perturbed by LatB treatment. 8-12 LBs were analyzed for each condition.

squeezing. In particular, myosin II is well known to associate with actin, which drives coat contraction (Nightingale et al., 2011; Yu and Bement, 2007b). Immunofluorescent staining of ATII cells fixed 2-3 minutes after stimulation revealed that myosin II is recruited to actin-coated LBs. All LBs that were coated with actin, as detected by phalloidin staining, were also positive for myosin II (Fig. 7A). Furthermore, treating ATII cells with myosin II inhibitor (-)-blebbistatin $(50 \mu \mathrm{M})$ significantly decreased the overall number of actin coats that contracted within 2 minutes after fusion, when $>96 \%$ of actin coats had contracted under control conditions (Fig. 7B). In (-)-blebbistatin-treated cells actin ring contraction was slowed down significantly (Fig. 7C). Treatment with (+)-blebbistatin, the inactive enantiomer of blebbistatin, had no significant effect on coat 
A

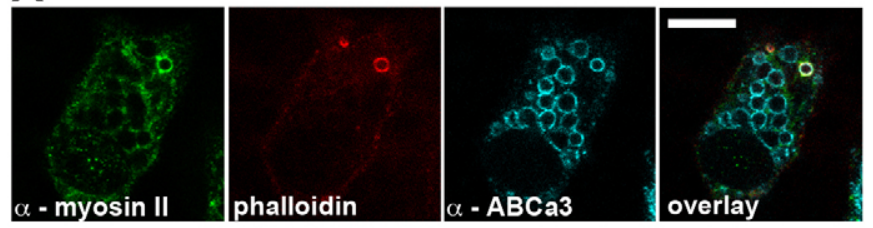

B

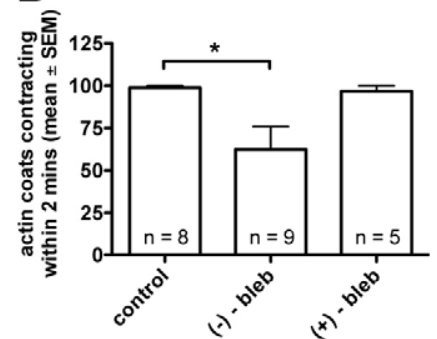

C

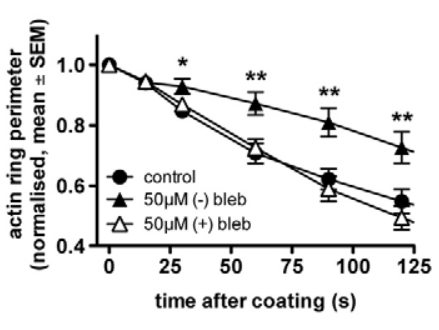

Fig. 7. Myosin II is recruited to actin coated LBs and facilitates coat compression. (A) Myosin II (green) colocalizes with actin coats (phalloidin, red) on LB membranes following fusion as detected by indirect immunofluorescence and confirmed by colocalization with P180 lamellar body protein (blue, $\mathrm{ABCa} 3$ ). Scale bar: $10 \mu \mathrm{m}$ (B) The number of actin coats starting to contract within 2 minutes following fusion is significantly reduced in cells treated with myosin II inhibitor (-)-blebbistatin but not $(+)$-blebbstatin, the negative enantiomer. $n$ represents number of experiments for each condition, up to 10 fusions were analyzed in each experiment. (C) (-)-blebbistatin slows down actin coat compression significantly. (+)-blebbstatin has no effect on actin coat compression. $>20$ actin coats were analyzed for each condition. ${ }^{*} P<0.05 ; * * P<0.01$.

compression. Although inhibition of myosin II had a significant effect on coat compression, ultimately most coats contracted even in the presence of (-)-blebbistatin, indicating that myosin II facilitates, but is not essential for, effective coat contraction. These results are similar to those observed for cortical granules in oocytes (Yu and Bement, 2007b) and Weibel-Palade bodies in endothelial cells (Nightingale et al., 2011).

\section{Inhibition of Rho and formins prevents actin coating of fused LBs}

It is still unclear what factors are necessary for actin coating of fused LBs. It has been shown that small GTPases of the Rho family regulate coating of secretory vesicles following exocytosis in oocytes and in pancreatic acinar cells (Nemoto et al., 2004; Sokac et al., 2003). To assess whether small GTPases are also required for coating of fused LBs, ATII cells were treated overnight with Clostridium difficile toxin B. Following incubation with toxin $\mathrm{B}$, the number of fused LBs coated with actin (see Fig. 8A) decreased significantly. In the presence of $100 \mathrm{nM}$ toxin $\mathrm{B},<14.0 \%$ of fusions were coated; when the concentration of toxin was increased to $300 \mathrm{nM}$, the fraction of coated LBs decreased to $<4 \% \quad(n=131$ and 58 fusions, respectively, $P<0.0001$ compared with untreated cells). To differentiate between the different classes of Rho GTPases, we next used inhibitors to selectively inhibit cdc42, Rho and Rac activity. Pharmacological inhibition of cdc42 (20 $\mu \mathrm{M}$ secramine) (Pelish et al., 2006) or Rac (100 $\mu \mathrm{M}$ NSC23766) showed no significant effect on actin coating of fused vesicles (Fig. 8A). However, when Rho GTPase activity was perturbed following incubation with $2 \mu \mathrm{g} / \mathrm{ml}$ of cell-permeable C3 transferase (Rho
A

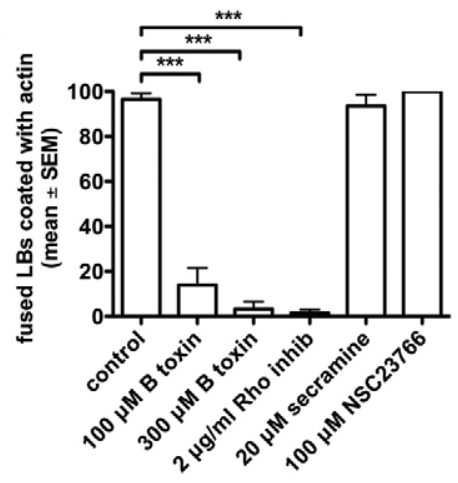

B

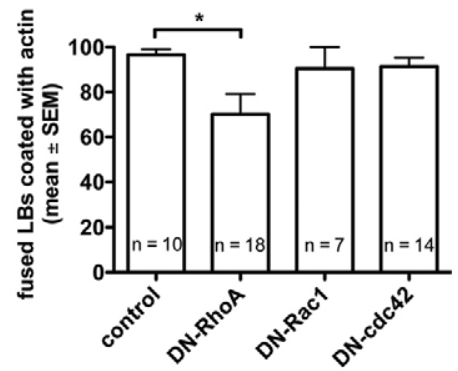

C

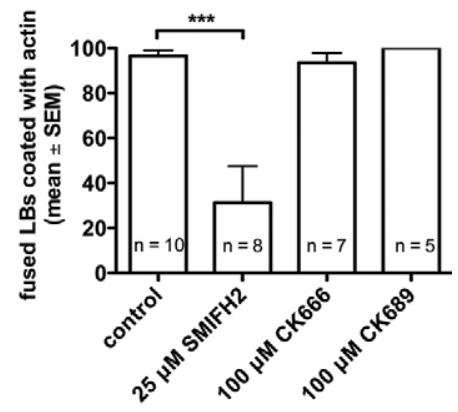

Fig. 8. Actin coat formation depends on Rho and formin. (A) Actin coating of fused LBs is almost completely inhibited following treatment with Clostridium difficile toxin B, an inhibitor for small GTPases. Specific inhibition was also observed with an inhibitor of Rho GTPases, but not inhibitors for cdc42 (secramine) or Rac1 (NSC23766). Data represent means \pm s.e.m. from $\geq 6$ experiments for each condition, up to 39 fusions were analyzed in each experiment. (B) Actin coating of fused LBs is significantly reduced in cells expressing DN-RhoA, but not in cells expressing DN-Rac1 or DN-cdc42. $n$ represents number of experiments for each condition, up to 28 fusions were analyzed in each experiment. (C) Actin coat formation on fused LBs was significantly reduced following treatment with formin inhibitor SMIFH2 (unpaired $t$-tests, $P<0.0004$ ), but not by inhibitor for Arp2/3 complex (CK666) or the negative control thereof (CK689). $n$ represents number of experiments for each condition, up to 26 fusions were analyzed in each experiment. $* P<0.05 ; * * P<0.005$.

Inhibitor), the fraction of fused LBs that were coated with actin was significantly reduced to $<2 \%(n=57$ fusions, $P<0.0001)$ (Fig. 8A). Similar results were observed in cells overexpressing dominant-negative (DN) mutants of cdc42, RhoA and Rac1. Genetic perturbation of cdc42 or Rac showed no significant effect on actin coating of fused vesicles. In cells overexpressing DN RhoA (T19N-RhoA) the fraction of fused LBs that were coated with actin was significantly reduced to $70.12 \pm 9.09 \%$ ( $n=128$ fusions) compared with $96.50 \pm 2.59 \%$ ( $n=76$ fusions) fused LBs that were coated under control conditions $(P=0.04$, Fig. 8B). 
Actin coating of fused LBs probably depends on nucleation of actin on fused vesicles. Treatment with LatB or jasplakinolide (Fig. 1B) prevents actin coating of LBs following fusion. It has been shown that Rho GTPases promote actin nucleation through activation of formins (Campellone and Welch, 2010). Treatment of ATII cells with formin inhibitor SMIFH2 (25 $\mu \mathrm{M})$ (Rizvi et al., 2009) significantly reduced the fraction of LBs that were coated with actin following fusion from $96.50 \pm 2.59 \%$ ( $n=76$ fusions) under control conditions to $31.25 \pm 16.19 \%$ ( $n=13$ fusions) $(P<0.0004$, Fig. 8C). We never observed obvious effects on cell viability (e.g. blebbing) by SMIFH2 (Rizvi et al., 2009). However, treatment with Arp2/3 inhibitor CK666 $(100 \mu \mathrm{M})$ had no effect on the formation of actin coats (Fig. 8C). Together, these results suggest that actin coating of fused vesicles depends on active Rho and that actin nucleation on fused vesicles is mediated by formins.

\section{Discussion}

We have recently shown that LB fusion with the PM is followed by selective actin coating of the fused LB. We have also observed that actin coating of fused vesicles precedes surfactant release (Miklavc et al., 2009b). Given the bulky, highly hydrophobic nature of the LB contents (surfactant), we hypothesized that active compression of vesicles by actin might be necessary for efficient secretion of surfactant by forced expulsion. However, direct proof for the requirement of actin coating of fused vesicles for surfactant secretion was still missing. Here, we demonstrate that actin coating of fused LBs is essential for active surfactant expulsion, and hence secretion. This is in accordance with a recent study reporting a similar actin-dependent function in release of the granule core protein von Willebrand factor from Weibel-Palade bodies in human endothelial cells (Nightingale et al., 2011). Similar to surfactant, von Willebrand factor constitutes a relatively large and bulky cargo that is not readily released from exocytic vesicles upon fusion pore opening (Babich et al., 2008). It is not surprising that additional mechanisms during the post-fusion phase are required for efficient secretion of such cargos. This can involve regulating the size of the initial fusion pore or fusion pore dilation (Albillos et al., 1997; Babich et al., 2008; Obermüller et al., 2005; Perrais et al., 2004; Tsuboi et al., 2004) or active extrusion of vesicle cargos. In ATII cells, fusion pores do expand in a $\mathrm{Ca}^{2+}$-dependent manner (Haller et al., 2001a; Miklavc et al., 2011), with most LBs exhibiting relatively large fusion pores that are stable for long periods (Haller et al., 2001a). This is in line with recent findings that fusion pores are the subjects of stabilization and that stable fusion pore diameters depend on vesicle sizes (Jorgačevski et al., 2010). However, despite their large size, fusion pore diameters of fused LBs were always significantly smaller than the vesicle diameters, and hence smaller than the bulky vesicle cargo complex (i.e. lamellar surfactant) (Haller et al., 2001a). Given the apparent stability of LB fusion pores, full widening into the PM is probably constrained. Hence, it is conceivable that fusion pores act as mechanical barriers for vesicle content release (Haller et al., 2001b; Singer et al., 2003) and additional mechanisms are required to promote efficient vesicle cargo discharge.

In the case of Weibel-Palade bodies, an actin filament ring acts as a minicytokinetic ring to exert force on the bottom of an open granule, pushing von Willebrand factor out on the other end into the extracellular environment (Babich et al., 2008). However the fate of the fused granule membrane in endothelial cells is not clear. The actin ring might travel along the fused vesicle, squeezing contents out of fused Weibel--Palade bodies, leaving empty granules ready for re-endocytosis, or the granule might collapse into the PM. In several non-mammalian systems, actin coat formation has been postulated as a mechanism for retrieval of empty cortical granules after they have released their contents (Becker and Hart, 1999; Sokac et al., 2003). However, we never observed compensatory endocytosis in ATII cells (Mair et al., 1999). A reason for this could be the low endocytic activity of these cells in our culture conditions. By directly and simultaneously imaging peri-vesicular actin and vesicle membrane dynamics, we found that actin forms a coat around fused vesicles, enclosing the entire vesicle, possibly also interacting or anchoring to the cortical actin near the site of fusion. Similar conclusions have been drawn from an elegant study looking at the secretory granules in the salivary glands of live animals (Masedunskas et al., 2011).

In ATII cells, extrusion is driven by compression of the whole vesicle, resulting in collapse of the vesicle into the PM. This finding suggests that the major function of peri-vesicular actin accumulation around LBs, as in endothelial cells, is the active expulsion of poorly soluble vesicle contents and not compensatory endocytosis. It still remains to be resolved how ATII cells retrieve the exocytosed vesicle membrane.

F-actin has also been shown to be involved in maintaining fusion pore open states of fused zymogen granules (Larina et al., 2007), probably in conjunction with myosin II (Bhat and Thorn, 2009). Similarly, regulation of fusion pore dilation by actin and myosin II in mouse adrenal chromaffin cells was found to control catecholamine and neuropeptide release (Doreian et al., 2008). We cannot fully exclude the possibility that actin coating of fused LBs also plays a role in maintaining an open fusion pore state, thereby facilitating surfactant secretion. In such a scenario, disruption of actin coats by LatB treatment would result in a narrowing or even closure of the fusion pore and result in the reduced surfactant expulsion or secretion observed in our experiments. However, we do not believe that regulation of fusion pore open state is the main regulatory function of actin coats in surfactant release. First, in contrast to zymogen granules where most fusion pores do eventually close under control conditions as a prelude to endocytosis (Larina et al., 2007), fusion pores of fused LBs very rarely close once opened (Haller et al., 2001a; Miklavc et al., 2009b), which suggests a slightly different mode of fusion pore regulation. Second, inhibition of actin polymerization (LatB) or myosin II [(-)-blebbistatin] does not have an impact on diffusion of fluorescent dyes across the fusion pore within several minutes of fusion pore opening. Quite the opposite, LatB treatment even facilitates diffusion of LTR from LBs upon fusion and fusion pore opening, indicating that at least at early times following fusion (up to 2 minutes), fusion pores are rather expanded when actin coat formation is inhibited (M.F., unpublished data). This is within the time frame when BODIPY-PC-labelled surfactant is expelled from most fused LBs (Fig. 2).

Regardless of the presumed function of the actin coat, its compression is probably modulated by myosin II (Masedunskas et al., 2011; Nightingale et al., 2011; Yu and Bement, 2007b). Myosin II is recruited to fused LBs following actin coating and inhibition of myosin II delays actin coat contraction. However, inhibition of myosin II does not completely block actin ring contraction, but rather delays it. This implies that myosin II is not essential for actin coat contraction, but seems to have a 
facilitating function. Whether myosin II is mainly and directly acting on the actin coat itself or whether it has additional functions is still unclear. As already outlined above, in pancreatic acinar cells, myosin II activity during the post-fusion phase is necessary to keep fusion pores open (Bhat and Thorn, 2009). We cannot rule out that myosin II recruited to fused LBs is also involved in regulation of the fusion pore dilation. Whether other myosins also play a role in regulating or modulating actin coat assembly and symmetry (Yu and Bement, 2007b) is the subject of ongoing studies. Alternatively, actin polymerization alone might be sufficient to compress the exocytic vesicle (Giardini et al., 2003; Sokac et al., 2003).

It is tempting to speculate what the benefit of such elaborate secretion machinery might be. Squeezing of surfactant through a narrow fusion pore (Haller et al., 2001a) might be essential for proper transformation of surfactant from a lamellar into a more tubular structure to facilitate insertion into the surfactant layer lining the hypophase (Goerke, 1998). Alternatively, prevention of the rapid release of surfactant might aid in sequential or selective release of various LB cargos (Tsuboi et al., 2004). Understanding the precise mechanisms that shape the secretory output might well aid the development of strategies to facilitate surfactant release under diseased conditions.

We have recently shown that actin coat formation on fused LBs is inhibited by chelation of cytoplasmic $\mathrm{Ca}^{2+}$ or phospholipase D inhibitor C2 ceramide (Miklavc et al., 2009b). Despite the dependence on $\mathrm{Ca}^{2+}$, actin coating was unaffected by FACE, 'fusion-activated' calcium entry (Miklavc et al., 2011) (supplementary material Fig. S1). We also speculated that actin coating might involve membrane mixing of PM and LB following fusion and follow a recently described mechanism involving protein kinase $\mathrm{C}$, cdc42 and neuronal Wiskott-Aldrich syndrome protein (N-WASP) (Yu and Bement, 2007a). Although we have evidence for membrane mixing [loss of lamp3-GFP from the LB and rapid diffusion of lyn-DsRed into the LB membrane upon fusion (Miklavc et al., 2009a)], we could not observe an effect of perturbing cdc 42 activity on actin coating. Moreover, we could not observe recruitment of N-WASP or an effect of the N-WASP inhibitor wiskostatin on actin coating.

Although our data do not exclude the possibility that actin filaments are translocated to the fused vesicles or that cortical actin engulfs them, none of these possibilities is likely. First, we did not observe any apparent translocation; second, actin coats appeared to form all around fused vesicles simultaneously, rather than sprouting from the site of fusion. We rather suggest de novo nucleation of actin filaments around fused LBs, similar to observations in PC12 cells (Gasman et al., 2004) or Xenopus oocytes (Yu and Bement, 2007a). However, in ATII cells, this is unlikely to be mediated by the Arp2/3 complex. We could not detect subunit Arp2 on fused LBs nor did treatment with Arp2/3 inhibitor impact actin coat formation. This is in line with recent observations in human endothelial cells (Nightingale et al., 2011). Our data rather suggest a dependence on active Rho and formins for actin coating. The exact mechanisms, therefore, remain unclear. We speculate that it involves activation of formins through Rho and subsequent actin nucleation. RhoA is suggested to activate formins (Campellone and Welch, 2010); however, our data do not exclude the idea that Rho proteins other than RhoA might be involved. Also, we have not yet identified the formin involved in this process. Regulation of actin coating of fused LBs could be analogous to a mechanism described in Salmonella invasion, where activation of a RhoA- and myosin-II-dependent but Arp2/3-independent pathway induces phagocytic cup formation (Hänisch et al., 2011).

In summary, our data demonstrate that LBs are coated with actin following fusion with the PM. This is probably dependent on activation of Rho and formin-dependent actin nucleation on fused vesicles. Subsequently, actin coats compress fused LBs, a process facilitated by myosin II. This compression is essential for active expulsion of surfactant and results in granule membrane flattening into the PM. Inhibition of actin coat formation resulted in strong reduction of surfactant secretion. Adding to the increasing evidence for regulatory mechanisms of secretion during the post-fusion phase, we postulate that regulation of fusion pore opening and dilation itself is not sufficient for release of bulky vesicle cargos and that active extrusion mechanisms are required.

\section{Materials and Methods \\ Materials}

All materials were purchased from Invitrogen (Karlsruhe, Germany) unless stated otherwise. Antibodies against myosin II and P180 lamellar body protein (ABCa3) were from Abcam (Cambridge, UK). Fluorescently labelled secondary antibodies were obtained from Molecular Probes (Invitrogen).

\section{Cell isolation}

ATII cells were isolated from Sprague-Dawley rats according to a published procedure (Dobbs et al., 1986) with minor modifications as recently described (Miklavc et al., 2010). After isolation, cells were seeded on glass coverslips, cultured in MucilAir (Epithelix, Switzerland), and used for experiments for up to 48 hours after isolation.

\section{Experimental conditions}

Experiments were performed as recently described (Miklavc et al., 2010). For all experiments, cells were kept in bath solution $(140 \mathrm{mM} \mathrm{NaCl}, 5 \mathrm{mM} \mathrm{KCl}, 1 \mathrm{mM}$ $\mathrm{MgCl}_{2}, 2 \mathrm{mM} \mathrm{CaCl}_{2}, 5 \mathrm{mM}$ glucose, $10 \mathrm{mM}$ HEPES, pH 7.4). 0.5-1 $\mu \mathrm{M}$ of FM 143 was added to the bath solution in experiments where LB fusion and surfactant secretion was detected by FM1-43 labelling (Haller et al., 1998). ATII cells were stimulated with $100 \mu \mathrm{M}$ ATP (Sigma, Schnelldorf, Germany). Inhibitors for actin polymerization (LatB, $10 \mu \mathrm{M}$ ) and myosin II (blebbistatin, $50 \mu \mathrm{M}$ ) were added 20 minutes and 2 hours before stimulation with ATP, respectively (all from Sigma, Schnelldorf, Germany). Inhibitors for small GTPases (B toxin), formins (SMIFH2) and Arp2/3 (CK666) were from Calbiochem (Darmstadt, Germany). Inhibitors for Rho (Rho Inhibitor) and Rac1 (NSC23766) were from Cytoskeleton (Denver, CO) and Tocris (Bristol, UK), respectively. For BODIPY-PC expulsion assays, cells were incubated overnight with $\beta$-BODIPY 500/510 C12-HPC $(1 \mu \mathrm{M})$ and preloaded for 15 minutes with LTR $(10-100 \mathrm{nM})$ before being washed twice in bath solution and kept in bath solution for the duration of the experiment. All fluorescent dyes were purchased from Molecular Probes (Invitrogen, Karlsruhe, Germany).

\section{Plasmids and adenoviruses}

Plasmids for lyn-GFP (Frick et al., 2007), GFP-actin and lamp-GFP (Miklavc et al., 2009b) were recently described, DsRed-actin was purchased from Clontech (TakaraBio, France) and GFP-cdc42(DN) (cdc42-T17N) was purchased from Addgene (clone 11400). Viruses were produced using the ViraPower Adenoviral Expression System (Invitrogen, Karlsruhe, Germany) according to the manufacturer's instructions. In brief, PCR products with attached attB sites were cloned into the entry vector pDONR221, using the BP Clonase II enzyme mix, and transferred from the entry vector into the adenoviral vector $\mathrm{pAd} / \mathrm{CMV} / \mathrm{V} 5$-DEST by in vitro recombination using LR Clonase. Expression plasmids were confirmed by sequencing and linearized using PacI before transfecting 293A cells. Cells were cultivated at $10 \% \mathrm{CO}_{2}$ and $95 \%$ humidity in DMEM medium containing $4.5 \mathrm{~g} / 1$ glucose, 10\% FBS, 1\% penicillin-streptomycin, and MEM nonessential amino acids for 5 to 8 days. For virus isolation, 293A cells were lysed by three freezethaw cycles (liquid nitrogen and $37^{\circ} \mathrm{C}$ ) and adenoviral particles were isolated using the Vivapure adenopack 10 virus isolation kit (Vivascience, Hannover, Germany), according to the manufacturer's protocol. Virus particles were aliquoted and stored at $-80^{\circ} \mathrm{C}$ in elution buffer containing $10 \%$ glycerol.

Adenoviruses expressing DN-RhoA (T19N-RhoA) and DN-Rac1 (T17N-Rac1) were a gift from Christopher M. Waters (University of Tennessee, Memphis, TN) (Desai et al., 2004). 


\section{Quantification of PC release}

PC release was quantified as recently described (Garcia-Verdugo et al., 2008). In short, an equal number of cells $\left(\sim 3.5 \times 10^{6}\right)$ were seeded into six-well plates. At day 2 after isolation, cells were washed three times in bath solution and preincubated in bath solution ( $\pm 10 \mu \mathrm{M}$ LatB) for 5 minutes before stimulation. The total volume in each well following stimulation was $1 \mathrm{ml}$. Cells were incubated for 15 minutes on a horizontal shaker $\left(\sim 120\right.$ r.p.m., $\left.37^{\circ} \mathrm{C}\right)$ before supernatants were collected and analyzed for PC content in triplicate for each sample using coupled enzymatic reactions based on a previous method to measure phospholipid in serum (Nanjee et al., 1991).

\section{AFM}

For combined AFM and FM1-43 measurements, an AFM system (model 5500, Agilent Technologies, Chandler, AZ) was mounted on an inverted optical microscope (IX81, Olympus) using an adapting sample stage. To reduce the effect of environmental vibration the AFM FM1-43 set-up was located on a vibration-damped table. For simultaneous recording of fluorescence images while imaging with AFM, a $610 \mathrm{~nm}$ long-pass glass filter (Thorlabs) was added to prevent interference of the fluorescence excitation radiation with the laser reflection onto the AFM photodiode. FM1-43 fluorescence was excited at $488 \mathrm{~nm}$ using Visichrome High Speed polychromator system (Visichrome, Germany) Fluorescence images were obtained using a cool snap EZ CCD camera (Visitron) and Metamorph software. We used a $40 \times$ LCPlanFI objective (Olympus) for fluorescence and bright-field images. Sequences of fluorescence images were taken while AFM imaging was performed to correlate the obtained optical images with AFM data. Cells were kept in bath solution for the duration of the experiments and investigated with AFM in either low force contact mode or dynamic (AAC) mode. AFM images were obtained using a large scanner $(100 \mu \mathrm{m}$ scan range) with scan rates of $0.3-0.5$ lines/second and a resolution of $512 \times 512$ pixels. V-shaped silicone nitride cantilevers with nominal spring constants ranging from $0.06-0.12 \mathrm{~N} / \mathrm{m}$ (manufacturer specifications, Veeco, Woodbury, NY) and a nominal tip radius of $30 \mathrm{~nm}$ were used. In both imaging modes, low force contact as well as AAC mode, the force set point was adjusted as low as possible to minimize the force exerted on the cell surface (typical forces were around 0.5 and $5 \mathrm{nN})$.

\section{Immunofluorescence}

For immunofluorescence staining, cells were washed twice in DPBS $(\mathrm{pH} 7.4$, Biochrom, Berlin, Germany) fixed for 20 minutes in $4 \%$ paraformaldehyde (Sigma) in DPBS and permeabilized for 10 minutes with $0.2 \%$ saponin and $10 \%$ FBS (Thermo Scientific, Bonn, Germany) in DPBS. Cells were subsequently stained with primary $(1: 300)$ and secondary $(1: 400)$ antibodies in PBS, $0.2 \%$ saponin and $10 \%$ FBS. Images were taken on an inverted confocal microscope (Leica TCS SP5, Leica, Germany) using a $63 \times$ lens (Leica HCX PL APO lambda blue $63.0 \times 1.40$ NA Oil UV). Images for the blue (DAPI), green (Alexa Fluor 488), red (Alexa Fluor 568) and far-red (Alexa Fluor 633) channels were taken in sequential mode using appropriate excitation and emission settings.

\section{Fluorescence imaging and photobleaching experiments}

Fluorescence imaging experiments were performed on a 2D imaging system (Visitron, Puchheim, Germany) and images were acquired using MetaFluor (Molecular Devices, Ismaning, Germany). Additionally, experiments were performed on a confocal imaging setup (Leica TCS SP5, Leica, Germany). To minimize channel crosstalk, appropriate filters were used for the 2D-imaging system and sequential mode acquisition for confocal imaging, respectively.

FM1-43 photobleaching experiments were performed on a Leica TCS SP5 confocal microscope with a $63 \times 1.4$ NA objective using the Leica FRAP Wizard software. All measurements were performed under the same conditions, with the pinhole set to 1.0 Airy unit and the confocal zoom set to 2 . A circle of interest was photobleached with ten scans with the $488 \mathrm{~nm}$ laser line at full power. Pre- and post-bleach images were monitored at low laser intensity for the times indicated.

\section{Image analysis and data presentation}

Images were analyzed using MetaFluor Analyst (Molecular Devices, Ismaning, Germany) and ImageJ (NIH, Bethesda, MD). MS Excel and GraphPad Prism 5 were used for statistics, curve fitting and graph design. Unless otherwise stated all data are presented as mean \pm s.e.m.

Actin coat contraction was analyzed by measuring the perimeter of individual actin rings at indicated time points after fusion. Similarly, changes in LB size following fusion were analyzed by measuring the perimeter of LBs, visible by expression of lamp3-GFP in the LB membrane or diffusion of lyn-DsRed into the LB membrane following fusion with the PM. To determine the onset of LB fusion, FM1-43 and LTR fluorescence was analyzed in a region encircling the fusing LB. Similarly, expulsion of surfactant was analyzed by measuring the decrease of BODIPY-PC fluorescence in a region encircling the fused LB. Only when BODIPY-PC fluorescence decreased $>10 \%$ following fusion, fusions were qualified as fusions with PC extrusion.
Analysis of the FM1-43 fluorescence recovery was performed by calculating the ratio between mean fluorescence intensity of the bleached LB and the mean fluorescence of an unbleached LB to correct for background bleaching during preand post-bleach acquisition. Ratio values were then expressed as a percentage of the prebleach ratio value. These normalized data were fitted to a single exponential curve by using the PRISM software (GraphPad Software, San Diego, CA) to derive $t_{1 / 2}$, the time at which half of the fluorescence has recovered.

\section{Acknowledgements}

We thank M. Timmler and T. Felder for technical assistance in particular for primary alveolar type II cell isolation and S. Britsch and C. Wiegreffe for access to and support for the Leica SP5 confocal microscope. We are grateful to C. M. Waters, Memphis, $\mathrm{TN}$, for providing the adenoviruses for expressing DN RhoA and rac1 and to T. Kirchhausen, Boston, MA, for providing secramine A, which came jointly from the Kirchhausen and the Hammond lab (University of Louisville) and was synthesized by B. Xu and G. B. Hammond.

\section{Funding}

This work was supported by the Deutsche Forschungsgemeinschaft [grant numbers D-1402/1-1 and D-1422/3-1] and the Landesstiftung Baden-Würtemberg [grant number P-LS-Biomat/03].

Supplementary material available online at

http://jcs.biologists.org/lookup/suppl/doi:10.1242/jcs.105262/-/DC1

\section{References}

Albillos, A., Dernick, G., Horstmann, H., Almers, W., Alvarez de Toledo, G. and Lindau, M. (1997). The exocytotic event in chromaffin cells revealed by patch amperometry. Nature 389, 509-512.

Albrecht, S., Usmani, S. M., Dietl, P. and Wittekindt, O. H. (2010). Plasma membrane trafficking in alveolar type II cells. Cell. Physiol. Biochem. 25, 81-90.

Anantharam, A., Bittner, M. A., Aikman, R. L., Stuenkel, E. L., Schmid, S. L., Axelrod, D. and Holz, R. W. (2011). A new role for the dynamin GTPase in the regulation of fusion pore expansion. Mol. Biol. Cell 22, 1907-1918.

Babich, V., Meli, A., Knipe, L., Dempster, J. E., Skehel, P., Hannah, M. J. and Carter, T. (2008). Selective release of molecules from Weibel-Palade bodies during a lingering kiss. Blood 111, 5282-5290.

Bean, A. J., Zhang, X. and Hökfelt, T. (1994). Peptide secretion: what do we know? FASEB J. 8, 630-638.

Becker, K. A. and Hart, N. H. (1999). Reorganization of filamentous actin and myosinII in zebrafish eggs correlates temporally and spatially with cortical granule exocytosis. J. Cell Sci. 112, 97-110.

Bhat, P. and Thorn, P. (2009). Myosin 2 maintains an open exocytic fusion pore in secretory epithelial cells. Mol. Biol. Cell 20, 1795-1803.

Campellone, K. G. and Welch, M. D. (2010). A nucleator arms race: cellular control of actin assembly. Nat. Rev. Mol. Cell Biol. 11, 237-251.

Desai, L. P., Aryal, A. M., Ceacareanu, B., Hassid, A. and Waters, C. M. (2004) RhoA and Rac1 are both required for efficient wound closure of airway epithelial cells. Am. J. Physiol. Lung Cell. Mol. Physiol. 287, L1134-L1144.

Dietl, P. and Haller, T. (2005). Exocytosis of lung surfactant: from the secretory vesicle to the air-liquid interface. Annu. Rev. Physiol. 67, 595-621.

Dietl, P., Haller, T., Mair, N. and Frick, M. (2001). Mechanisms of surfactant exocytosis in alveolar type II cells in vitro and in vivo. News Physiol. Sci. 16, 239243.

Dobbs, L. G., Gonzalez, R. and Williams, M. C. (1986). An improved method for isolating type II cells in high yield and purity. Am. Rev. Respir. Dis. 134, 141-145.

Doreian, B. W., Fulop, T. G. and Smith, C. B. (2008). Myosin II activation and actin reorganization regulate the mode of quantal exocytosis in mouse adrenal chromaffin cells. J. Neurosci. 28, 4470-4478.

Eitzen, G. (2003). Actin remodeling to facilitate membrane fusion. Biochim. Biophys. Acta 1641, 175-181.

Frick, M., Eschertzhuber, S., Haller, T., Mair, N. and Dietl, P. (2001). Secretion in alveolar type II cells at the interface of constitutive and regulated exocytosis. Am. J. Respir. Cell Mol. Biol. 25, 306-315.

Frick, M., Schmidt, K. and Nichols, B. J. (2007). Modulation of lateral diffusion in the plasma membrane by protein density. Curr. Biol. 17, 462-467.

Garcia-Verdugo, I., Ravasio, A., de Paco, E. G., Synguelakis, M., Ivanova, N., Kanellopoulos, J. and Haller, T. (2008). Long-term exposure to LPS enhances the rate of stimulated exocytosis and surfactant secretion in alveolar type II cells and upregulates P2Y2 receptor expression. Am. J. Physiol. Lung Cell. Mol. Physiol. 295, L708-L717.

Gasman, S., Chasserot-Golaz, S., Malacombe, M., Way, M. and Bader, M. F. (2004). Regulated exocytosis in neuroendocrine cells: a role for subplasmalemmal Cdc42/N-WASP-induced actin filaments. Mol. Biol. Cell 15, 520-531. 
Giardini, P. A., Fletcher, D. A. and Theriot, J. A. (2003). Compression forces generated by actin comet tails on lipid vesicles. Proc. Natl. Acad. Sci. USA 100, 64936498

Goerke, J. (1998). Pulmonary surfactant: functions and molecular composition. Biochim. Biophys. Acta 1408, 79-89.

Haller, T., Ortmayr, J., Friedrich, F., Völkl, H. and Dietl, P. (1998). Dynamics of surfactant release in alveolar type II cells. Proc. Natl. Acad. Sci. USA 95, 1579-1584.

Haller, T., Dietl, P., Pfaller, K., Frick, M., Mair, N., Paulmichl, M., Hess, M. W., Furst, J. and Maly, K. (2001a). Fusion pore expansion is a slow, discontinuous, and $\mathrm{Ca} 2+-$ dependent process regulating secretion from alveolar type II cells. J. Cell Biol. $155,279-290$

Haller, T., Pfaller, K. and Dietl, P. (2001b). The conception of fusion pores as ratelimiting structures for surfactant secretion. Comp. Biochem. Physiol. A Mol. Integr. Physiol. 129, 227-231.

Hänisch, J., Kölm, R., Wozniczka, M., Bumann, D., Rottner, K. and Stradal, T. E. (2011). Activation of a RhoA/myosin II-dependent but Arp $2 / 3$ complex-independent pathway facilitates Salmonella invasion. Cell Host Microbe 9, 273-285.

Hecht, E., Usmani, S. M., Albrecht, S., Wittekindt, O. H., Dietl, P., Mizaikoff, B. and Kranz, C. (2011). Atomic force microscopy of microvillous cell surface dynamics at fixed and living alveolar type II cells. Anal. Bioanal. Chem. 399, 23692378

Heuser, J. E. and Reese, T. S. (1973). Evidence for recycling of synaptic vesicle membrane during transmitter release at the frog neuromuscular junction. J. Cell Biol. 57, 315-344.

Jackson, M. B. and Chapman, E. R. (2008). The fusion pores of Ca2+-triggered exocytosis. Nat. Struct. Mol. Biol. 15, 684-689.

Jorgačevski, J., Fosnaric, M., Vardjan, N., Stenovec, M., Potokar, M., Kreft, M., Kralj-Iglic, V., Iglic, A. and Zorec, R. (2010). Fusion pore stability of peptidergic vesicles. Mol. Membr. Biol. 27, 65-80.

Jorgačevski, J., Potokar, M., Grilc, S., Kreft, M., Liu, W., Barclay, J. W., Bückers, J., Medda, R., Hell, S. W., Parpura, V. et al. (2011). Munc18-1 tuning of vesicle merger and fusion pore properties. J. Neurosci. 31, 9055-9066.

Larina, O., Bhat, P., Pickett, J. A., Launikonis, B. S., Shah, A., Kruger, W. A., Edwardson, J. M. and Thorn, P. (2007). Dynamic regulation of the large exocytotic fusion pore in pancreatic acinar cells. Mol. Biol. Cell 18, 3502-3511.

Lindau, M. and Gomperts, B. D. (1991). Techniques and concepts in exocytosis: focus on mast cells. Biochim. Biophys. Acta 1071, 429-471.

Mair, N., Haller, T. and Dietl, P. (1999). Exocytosis in alveolar type II cells revealed by cell capacitance and fluorescence measurements. Am. J. Physiol. 276, L376-L382.

Masedunskas, A., Sramkova, M., Parente, L., Sales, K. U., Amornphimoltham, P., Bugge, T. H. and Weigert, R. (2011). Role for the actomyosin complex in regulated exocytosis revealed by intravital microscopy. Proc. Natl. Acad. Sci. USA 108, 1355213557.

Miklave, P., Albrecht, S., Wittekindt, O. H., Schullian, P., Haller, T. and Dietl, P. (2009a). Existence of exocytotic hemifusion intermediates with a lifetime of up to seconds in type II pneumocytes. Biochem. J. 424, 7-14.

Miklave, P., Wittekindt, O. H., Felder, E. and Dietl, P. (2009b). Ca2+-dependent actin coating of lamellar bodies after exocytotic fusion: a prerequisite for content release or kiss-and-run. Ann. N. Y. Acad. Sci. 1152, 43-52.

Miklave, P., Frick, M., Wittekindt, O. H., Haller, T. and Dietl, P. (2010). Fusionactivated $\mathrm{Ca}(2+)$ entry: an "active zone" of elevated $\mathrm{Ca}(2+)$ during the postfusion stage of lamellar body exocytosis in rat type II pneumocytes. PLOS ONE 5, e10982.

Miklave, P., Mair, N., Wittekindt, O. H., Haller, T., Dietl, P., Felder, E., Timmler, M. and Frick, M. (2011). Fusion-activated Ca2+ entry via vesicular P2X4 receptors promotes fusion pore opening and exocytotic content release in pneumocytes. Proc. Natl. Acad. Sci. USA 108, 14503-14508.

Nanjee, M. N., Gebre, A. K. and Miller, N. E. (1991). Enzymatic fluorometric procedure for phospholipid quantification with an automated microtiter plate fluorometer. Clin. Chem. 37, 868-874.

Nemoto, T., Kojima, T., Oshima, A., Bito, H. and Kasai, H. (2004). Stabilization of exocytosis by dynamic F-actin coating of zymogen granules in pancreatic acini. J. Biol. Chem. 279, 37544-37550.
Nightingale, T. D., White, I. J., Doyle, E. L., Turmaine, M., Harrison-Lavoie, K. J., Webb, K. F., Cramer, L. P. and Cutler, D. F. (2011). Actomyosin II contractility expels von Willebrand factor from Weibel-Palade bodies during exocytosis. J. Cell Biol. 194, 613-629.

Obermüller, S., Lindqvist, A., Karanauskaite, J., Galvanovskis, J., Rorsman, P. and Barg, S. (2005). Selective nucleotide-release from dense-core granules in insulinsecreting cells. J. Cell Sci. 118, 4271-4282.

Pelish, H. E., Peterson, J. R., Salvarezza, S. B., Rodriguez-Boulan, E., Chen, J. L., Stamnes, M., Macia, E., Feng, Y., Shair, M. D. and Kirchhausen, T. (2006). Secramine inhibits Cdc42-dependent functions in cells and Cdc42 activation in vitro. Nat. Chem. Biol. 2, 39-46.

Perrais, D., Kleppe, I. C., Taraska, J. W. and Almers, W. (2004). Recapture after exocytosis causes differential retention of protein in granules of bovine chromaffin cells. J. Physiol. 560, 413-428.

Ravasio, A., Hobi, N., Bertocchi, C., Jesacher, A., Dietl, P. and Haller, T. (2011). Interfacial sensing by alveolar type II cells: a new concept in lung physiology? Am. J. Physiol. Cell Physiol. 300, C1456-C1465.

Rettig, J. and Neher, E. (2002). Emerging roles of presynaptic proteins in $\mathrm{Ca}++-$ triggered exocytosis. Science 298, 781-785.

Rizvi, S. A., Neidt, E. M., Cui, J., Feiger, Z., Skau, C. T., Gardel, M. L., Kozmin, S. A. and Kovar, D. R. (2009). Identification and characterization of a small molecule inhibitor of formin-mediated actin assembly. Chem. Biol. 16, 1158-1168.

Rizzoli, S. O. and Jahn, R. (2007). Kiss-and-run, collapse and 'readily retrievable' vesicles. Traffic 8, 1137-1144

Schafer, D. A. (2003). Actin puts on the squeeze. Nat. Cell Biol. 5, 693-694.

Singer, W., Frick, M., Haller, T., Bernet, S., Ritsch-Marte, M. and Dietl, P. (2003). Mechanical forces impeding exocytotic surfactant release revealed by optical tweezers. Biophys. J. 84, 1344-1351.

Soekmadji, C. and Thorn, P. (2010). Secretory control: evidence for agonist regulation of post-fusion vesicle behaviour. Clin. Exp. Pharmacol. Physiol. 37, 218-221.

Sokac, A. M. and Bement, W. M. (2006). Kiss-and-coat and compartment mixing: coupling exocytosis to signal generation and local actin assembly. Mol. Biol. Cell 17, 1495-1502.

Sokac, A. M., Co, C., Taunton, J. and Bement, W. (2003). Cdc42-dependent actin polymerization during compensatory endocytosis in Xenopus eggs. Nat. Cell Biol. $\mathbf{5}$, 727-732.

Südhof, T. C. (2004). The synaptic vesicle cycle. Annu. Rev. Neurosci. 27, 509-547.

Tsilibary, E. C. and Williams, M. C. (1983a). Actin and secretion of surfactant. J. Histochem. Cytochem. 31, 1298-1304.

Tsilibary, E. C. and Williams, M. C. (1983b). Actin in peripheral rat lung: S1 labeling and structural changes induced by cytochalasin. J. Histochem. Cytochem. 31, 12891297.

Tsuboi, T., McMahon, H. T. and Rutter, G. A. (2004). Mechanisms of dense core vesicle recapture following "kiss and run" ("cavicapture") exocytosis in insulinsecreting cells. J. Biol. Chem. 279, 47115-47124.

Turvey, M. R. and Thorn, P. (2004). Lysine-fixable dye tracing of exocytosis shows Factin coating is a step that follows granule fusion in pancreatic acinar cells. Pflugers Arch. 448, 552-555.

Valentijn, J. A., Valentijn, K., Pastore, L. M. and Jamieson, J. D. (2000). Actin coating of secretory granules during regulated exocytosis correlates with the release of rab3D. Proc. Natl. Acad. Sci. USA 97, 1091-1095.

van Weeren, L., de Graaff, A. M., Jamieson, J. D., Batenburg, J. J. and Valentijn, J. A. (2004). Rab3D and actin reveal distinct lamellar body subpopulations in alveolar epithelial type II cells. Am. J. Respir. Cell Mol. Biol. 30, 288-295.

Vardjan, N., Stenovec, M., Jorgačevski, J., Kreft, M. and Zorec, R. (2007). Subnanometer fusion pores in spontaneous exocytosis of peptidergic vesicles. J. Neurosci. 27, 4737-4746.

Yu, H. Y. and Bement, W. M. (2007a). Control of local actin assembly by membrane fusion-dependent compartment mixing. Nat. Cell Biol. 9, 149-159.

Yu, H. Y. and Bement, W. M. (2007b). Multiple myosins are required to coordinate actin assembly with coat compression during compensatory endocytosis. Mol. Biol. Cell 18, 4096-4105. 